University of Warwick institutional repository: http://go.warwick.ac.uk/wrap This paper is made available online in accordance with publisher policies. Please scroll down to view the document itself. Please refer to the repository record for this item and our policy information available from the repository home page for further information.

To see the final version of this paper please visit the publisher's website. Access to the published version may require a subscription.

Author(s): David Tall

Article Title: The notion of infinite measuring number and its relevance in the intuition of infinity Year of publication: 1980

Link to published version: http://dx.doi.org/ 10.1007/BF00697740

Publisher statement: The original publication is available at www.springerlink.com 


\title{
The Notion of Infinite Measuring Number and Its Relevance in the Intuition of Infinity
}

\author{
David Tall \\ Mathematics Education Research Centre, \\ University of Warwick, \\ COVENTRY CV4 7AL, UK.
}

\begin{abstract}
In this paper a concept of infinity is described which extrapolates the measuring properties of number rather than counting aspects (which lead to cardinal number theory).

Infinite measuring numbers are part of a coherent number system extending the real numbers, including both infinitely large and infinitely small quantities, A suitable extension is the superreal number system described here; an alternative extension is the hyperreal number field used in non-standard analysis which is also mentioned.

Various theorems are proved in careful detail to illustrate that certain properties of infinity which might be considered 'false' in a cardinal sense are 'true' in a measuring sense, Thus cardinal infinity is now only one of a choice of possible extensions of the number concept to the infinite case, It is therefore inappropriate to judge the 'correctness' of intuitions of infinity within a cardinal framework alone, especially those intuitions which relate to measurement rather than one-one correspondence.

The same comments apply in general to the analysis of naive intuitions within an extrapolated formal framework.
\end{abstract}

\section{Introduction}

Numbers are used for at least three basic purposes in everyday life counting, ordering and measuring. At the end of the last century Cantor extended the first two of these by introducing infinite cardinals and ordinals and such interpretations have become well accepted by our formal mathematical culture. However there are occasions when they are not the most appropriate extension of the number concept; in particular, where measurement is involved an infinite interpretation of measuring number may be more suitable.

An example will make this more apparent. Suppose we consider two line segments $A B, C D$ where $C D$ is twice as long as $A B$ (Figure 1) and ask if there are the same number of points in $A B$ and $C D$. 

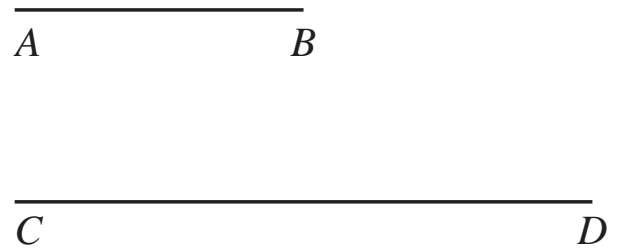

Figure 1

The cardinal number solution is that there is a correspondence between the two sets of points in which a point $P$ on $A B$ distance $x$ from $A$ corresponds to a point $Q$ on $C D$ distance $2 x$ from $C$, so they have the same cardinal number (Figure 2).

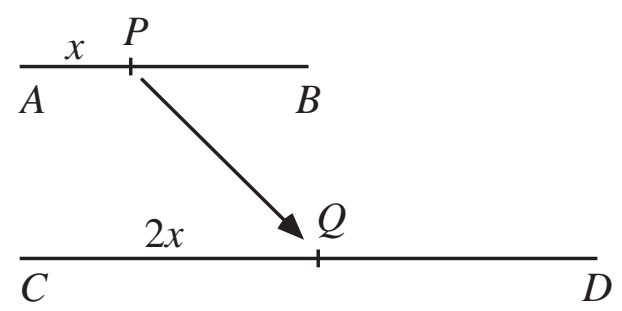

Figure 2

A perfectly reasonable alternative is that $C D$ has twice as many points as $C D$. The cardinal response to this (see [5, p. 244]) is that if the set of points in $A B$ has cardinality then those in $C D$ do have cardinality 2 , but this may only confuse the issue, for it conflicts with the reasonable intuition that twice some positive quantity must be strictly larger than that quantity.

The usual response to this conflict is a re-education of the individual so that he or she learns to accept that such phenomena naturally occur with infinite cardinals, indeed it is the property of a set being put in one-one correspondence with a proper subset which precisely characterises cardinal infinities. Such logical sleight of hand does not always satisfy the cognitive psychological requirements of the learner.

The existence of various intuitions of infinity has been extensively investigated by Fischbein (for example in [I] and [2]). It was through reading his work and having informal discussions with him that this paper came to be written. to provide a basis for an alternative interpretation of children's intuitions.

If we consider the intuitive notion of 'point' and 'line' we shall find that an alternative interpretation of infinity is possible without the 
conflicts that occur in this example. The reason that one supposes that there are twice as many points in $C D$ as $A B$ is because measurement is involved rather than counting.

Physical points have size when they are marked with the stroke of a pen on a picture. so that the longer the interval, the larger the number of points that can be fitted in. Children have many different intuitions as to the nature of a point. One may not 'see' points on a line segment until they are marked in, another may say that a line segment has four points (one at each corner), and so on. At some stage they are introduced to the mathematical convention of marking a point on a line with a pen stroke as in Figure 2. At this stage 'points' may be interpreted by nonoverlapping marks and when asked to mark as many points as possible in an interval a child may do so until he can fit no more marks physically within the gaps left. The same child, shown two points on a piece of paper and asked to mark all the points on a straight line between them, may then draw overlapping blobs in a line joining the given points. The blobs can still be seen to be distinct entities, but the overlaps are necessary to manufacture a connected line between the points given at the outset.

This may suggest that a child at this stage believes in a "finitistic" line, made up of a finite number of indivisible points, but that is not true. The same child (a boy of eight years) was asked "If we take a line as long as the road outside and cut it in half, then take half of one of the pieces, and then half of one of these, and on and on, can we go on for ever, or do we eventually have to stop?" He replied "You can go on for ever." "But won't the pieces eventually get too small to cut in half?" "No. not if you look through a microscope."

One may postulate that such a child is in some kind of Piagetian transition stage between finite and infinite divisibility of a line. An alternative hypothesis is that the child has come up against the genuine conflict between finite divisibility in practical drawing and infinite divisibility in theoretical imagination. What is absolutely clear is that the child does not conceive the notion of number in a cardinal sense. He sees it as a crude kind of measurement. "How many points can you imagine on that line?" "About fifty." "And ..." (pointing to a line twice the length) "how many on that line?" "A hundred." The number of points on a line is proportional to the length of the line for him. He has no conception of the difference between the rational continuum and the real 
continuum which have different cardinal numbers, nor the fact that real intervals of different length have the same cardinal number. It is therefore totally inappropriate to interpret his thinking processes within a formal cardinal paradigm.

There is a genuine problem here. If we do not use a cardinal interpretation, what can we use, for surely any formal interpretation is not a true description of the intuition of the child. A partial answer to this problem is to provide an alternative formal interpretation that is logically complete and yet is consonant with the child's intuition in places which prove conflicting with a cardinal interpretation. By this process we can at least realise the relative nature of our interpretation. The cardinal paradigm is then seen as only one of several possible infinite extensions of the number concept rather than an absolute logical reality against which everything must be judged.

\section{Theoretical 'points' with size}

Points marked with a pen in a physical picture have a finite size and only a finite number of them are required to cover an interval of finite length. To uniformize the theory, let us suppose that the pen strokes have thickness $d$, then we shall need (approximately) $l / d$ of them to cover an interval of length $l$. Here we are using number in a measuring sense. By analogy, let us consider the answer to the question "how many inches in a metre?" The answer is a real number which is not an integer. More generally, if we ask how many intervals length $d$ are required to cover an interval length $l$, the answer may be interpreted as the real number $l / d$. We now suppose that a 'point' is a (closed) interval of length $d$. Of course, only a finite number are required to cover a finite interval. However, if we take $d$ to be an infinitesimal, then $l / d$ will then be an infinite number, not an infinite cardinal, but an infinite measuring number. If we take an interval of twice the length, $2 l$, then the number of "points" in the interval is $2 / / d$, which is precisely twice as many.

To explain such a theory requires a formal interpretation of the notion of an infinitesimal. This may be done using non-standard analysis [4] but may be performed in a much simpler way by introducing a single infinitesimal. We shall explain the latter in $\$ 3$ by describing the superreal numbers of [6] which are the real numbers expanded to include power series in an infinitesimal $\varepsilon$. If a 'point' has infinitesimal size, say $\varepsilon$, we discover a theory which allows both indivisibility of 
'points' and also infinite divisibility of a line (in sharp contrast to the classical Greek dichotomy which only envisaged one or the other). These factors are considered in $\$ 4$ before computing the cardinal number of such 'points' in an interval in $\$ 5$. The main thrust of the paper is reached with infinite measuring numbers in $\$ 6$ and a brief reference to a non-standard interpretation in \$7. Having laid the groundwork, we close by looking at the intuition of infinity and see how our perception is distorted by a mathematical culture which accepts infinite cardinals but has little acquaintance with infinitesimals and the infinite measuring number concept.

\section{The superreals}

In modern mathematics the number line is usually interpreted in terms of the real number system where each number has a decimal expansion

$$
a_{-m} 10^{m}+\ldots+a_{-1} 10+a_{0}+a_{1} 10^{-1}+\ldots+a_{n} 10^{-n}+\ldots
$$

with the coefficients $a_{i}$ being digits between 0 and 9 . In such a system there may seem no room to introduce infinitesimals. But prior to the nineteenth century the number line was often visualised as including infinitesimally small quantities. A modern description of such a system can be given by considering power series expansions analogous to decimals. The superreal numbers $\Re$ are defined to be power series in an indeterminate $\varepsilon$ of the form

$$
a_{-m} \varepsilon^{-m}+\ldots+a_{-1} \varepsilon^{-1}+a_{0}+a_{1} \varepsilon+\ldots+a_{n} \varepsilon^{n}+\ldots
$$

where each coefficient $a_{r}$ can be any real number whatsoever. The superreals can be thought of as 'epsimal' expansions where addition and multiplication are performed according to the usual rules for power series. They include the real numbers as elements where $a_{r}=0$ for $r \neq 0$ and can be given the structure of an ordered field by defining a nonzero element

$$
\alpha=\sum_{n=k}^{\infty} a_{n} \varepsilon^{n},\left(a_{k} \neq 0\right)
$$

to be 'positive' if $a_{k}>0$. For instance both $\varepsilon$ and $a-\varepsilon$ (for positive real $a$ ) are 'positive'. If we now write ' $\alpha>\beta$ ' or ' $\beta>\alpha$ ' instead of ' $\alpha-\beta$ ' is positive', then we find

$$
0<\varepsilon<a \text { for every positive } a \in \boldsymbol{R}
$$

so $\varepsilon$ is a positive infinitesimal.

The system of superreal numbers is described in detail in [7]. In general, superreals of the form $\alpha=\sum_{n=k}^{\infty} a_{n} \varepsilon^{n}$ are infinitesimal when $k>0$ and infinite when $k<0$. A finite element is of the form $\alpha=\sum_{n=0}^{\infty} a_{n} \varepsilon^{n}$. 
If we wish to visualize such expressions as points on a number line, then infinite elements such as $-1 / \varepsilon$ or $1 / \varepsilon$ are so far off to the left or right that they cannot be drawn on a finite diagram. Meanwhile, all that can be seen of a finite element $\sum_{n=0}^{\infty} a_{n} \varepsilon^{n}$ is its standard part $\operatorname{st}(\alpha)=a_{0}$, because the remainder of the expansion is infinitesimal. For instance, st $(\varepsilon)=0$ so the difference between $\varepsilon$ and 0 cannot be represented in a normal-scale diagram (Figure 3).

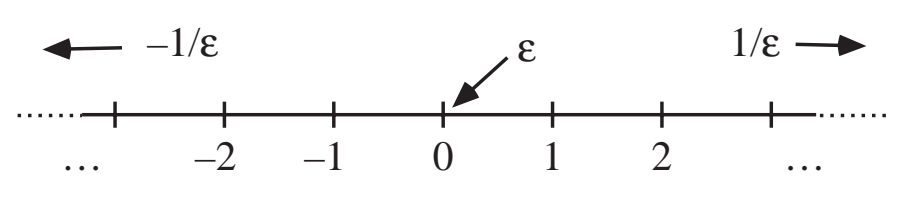

Figure 3

However, the map, $\mu: \Re \rightarrow \Re$ in which $\mu(x)=(x-\alpha) / \delta$ shifts $\alpha$ to the origin and divides by the scale factor $\delta$. By judicious choice of $\alpha$ and $\delta$ (as infinite or infinitesimal elements), details can be revealed which are not visible to normal scale. For instance, if $\alpha=a$ is real and $\delta=\varepsilon$, then points of the form $a+\lambda \varepsilon(\lambda \in \boldsymbol{R})$ which are infinitesimally close to $a$ and to each other are mapped onto distinct points $\lambda \in \boldsymbol{R}$. By such a device we can 'see' infinitesimal detail. A full description of such techniques is given in [7].

\section{Indivisibles}

Returning to ordinary pen drawings on paper for the moment, we note that we cannot distinguish between points which are very close together. A fine pen draws a line 0.1 millimetres thick, so if we take a unit length to be, say, 10 centimetres, then a pen-stroke is $10^{-3}$ units wide, so we certainly cannot distinguish between $\pi$ and 3.1416 whose difference is less than $10^{-4}$, or one tenth of a pen-stroke.

To model this we choose a positive number $d$ and say that two numbers $a, b$ are $d$-indistinguishable if

$$
-d \leq a-b \leq d \text {. }
$$

We then define a subset $P$ of real (or superreal) numbers to be a $d$ indivisible if all pairs of elements of $P$ and $d$-indistinguishable. A $d$ indivisible is, at its largest, a closed interval of length $d$. For finite $d$ it doesn't really matter whether we regard the underlying system to be 
rational, real or superreal in the sense that if we ask how many $d$ indivisibles are required to cover an interval length $l$, then the answer is $l / d$. If $l$ and $d$ are rational, we get the same answer whichever underlying continuum we consider. Notice also that the continuum (whichever we use) is only finitely divisible in the sense that we can break it down into a finite number of indivisibles which are then so small that the points within them are indistinguishable.

Now suppose that $d$ is infinitesimal, which means we require a continuum with infinitesimals. One obvious choice is to work with the superreal continuum and, for $a, b \in \mathfrak{R}(a<b)$, consider the interval

$$
[a, b]^{\#}=\{x \in \mathfrak{R} \mid a \leq x \leq b\} .
$$

This is very much a Leibnizian continuum in the sense that it includes all points of the form $a+\delta, b-\delta$, and $c \pm \delta$ where $\delta$ is a positive infinitesimal and $a<c<b$. (We would do well to remember that prior to the nineteenth century a line segment was considered to include infinitesimals as well.) To obtain an analogy with the rational continuum, instead of dealing with the whole field of superreals, we could start with the rational numbers $\boldsymbol{Q}$ and adjoin only arithmetic combinations of the infinitesimal $\varepsilon$. This means working in the field $\boldsymbol{Q}(\varepsilon)$ of rational functions in $\varepsilon$. An element in this field is of the form

$$
\frac{a_{0}+a_{1} \varepsilon+\ldots+a_{n} \varepsilon^{n}}{b_{0}+b_{1} \varepsilon+\ldots+b_{m} \varepsilon^{m}}\left(a_{i}, b_{j} \in \boldsymbol{Q}\right) \text {. }
$$

Working only with elements of this form, we get a 'rational continuum with infinitesimals which has closed intervals of the form

$$
[a, b]^{+}=\{x \in Q(\varepsilon) \mid a \leq x \leq b\} .
$$

If we cover either of these continua with $\delta$-indivisibles for infinitesimal $\delta$, then clearly we shall need an infinite number to cover a finite interval. We may interpret this infinite number as a cardinal number or a measuring number. In doing so we shall find that the behaviour of the two types of infinity are quite distinct. We begin with the cardinal infinity in the next section.

\section{Some cardinality considerations}

First we compute the cardinal number of the set of superreals. Surprisingly, although we have added many infinitesimals and infinite elements to $\boldsymbol{R}$ to get $\mathfrak{R}$, the cardinal number is not actually increased: 
THEOREM 1 . The cardinality of the superreal numbers $\mathfrak{R}$ is $\boldsymbol{N}$, the same as that of the real numbers $\boldsymbol{R}$.

The proof of this result is given in an appendix. As a corollary, it is a straightforward matter to deduce:

THEOREM 2. The cardinality of any superreal interval

$$
[a, b]^{\#}=\{x \in \Re \mid a \leq x \leq b\} \quad(a<b)
$$

is $\aleph$.

Suppose we consider an interval $[a, b]^{\#}$ of finite length (with $a, b \in \boldsymbol{R}$ ) and consider how many intervals of infinitesimal length $\delta$ are needed to cover it. For any real $c$ in $a \leq c \leq b$ and any integer $n$ the interval

$$
I_{c, n}=[c+n \varepsilon, c+(n+1) \varepsilon]
$$

is of length $\varepsilon$. Every $\alpha \in[a, b]^{\#}$ is of the form

$$
\alpha=a_{0}+a_{1} \varepsilon+\ldots
$$

where $a \leq a_{0} \leq b$ and $n \leq a_{1} \leq n+1$ (for some integer $n$ ), so

$$
\alpha \in I_{a_{0}, n}
$$

Thus the intervals $I_{c, n}(a \leq c \leq b, n \in Z)$ cover $[a, b]^{\#}$ and the cardinality of this set of intervals is $\boldsymbol{\aleph} \times \boldsymbol{\aleph}_{0}=\boldsymbol{\aleph}$. Since each infinitesimal interval can contain at most one real number, this is the minimum cardinality that will be successful, giving:

THEOREM 3. The minimum cardinality of a set $S$ of intervals of length $\varepsilon$ required to cover $[a, b]^{\#}(a, b \in \boldsymbol{R}, a<b)$ is $\boldsymbol{\aleph}$.

A generalization of this result is:

THEOREM 4. If $\delta$ is a positive infinitesimal and $a, b$ are real numbers ( $a$ $<b$ ), the minimum cardinality of a set $S$ of intervals length $\delta$ required to cover $[a, b]^{\#}$ is $\boldsymbol{\aleph}$.

Similar computations can be made for the field $Q(\varepsilon)$ of 'rational infinitesimals' to obtain the following results:

THEOREM 5. The cardinality of $\boldsymbol{Q}(\varepsilon)$ is $\boldsymbol{\aleph}_{0}$.

THEOREM 6. For rational numbers $a$ and $b(a<b)$, the rational interval with infinitesimals $[a, b]^{+}=\{x \in \boldsymbol{Q}(\varepsilon) \mid a<b\}$ has cardinality $\boldsymbol{\aleph}_{0}$. 
THEOREM 7. For any positive infinitesimal $\delta \in \boldsymbol{Q}(\varepsilon)$, the minimum cardinality of a set $S$ of intervals length $\delta$ required to cover $[a, b]^{+}(a, b \in$ $Q, a<b)$ is $\boldsymbol{\aleph}_{0}$.

The proofs of these results are the same as those for Theorems 1-4, with rational numbers replacing real numbers and every appearance of $\boldsymbol{\aleph}$ replaced by $\boldsymbol{\aleph}_{0}$. The net result of all these cardinality computations is that, if we consider the number of infinitesimal indivisibles needed to cover a finite interval, then a superreal interval requires as many as there are real numbers in that interval, whilst a 'rational interval with infinitesimals' requires as many as there are rational numbers in that interval. The length of the interval, or the size of the infinitesimal indivisibles, on the other hand, is of no account.

\section{Infinite measuring numbers}

Now let us consider the number of $\delta$-indivisibles in an interval $[a, b]^{+}$or $[a, b]^{\#}$ from a measuring viewpoint. If the interval is length $l=b-a$, then the (measuring) number of $\delta$-indivisibles required to cover it is defined to be the superreal number $l / \delta$.

Notice that this is independent of the underlying continuum (provided that $l, \delta \in Q(\varepsilon)$ ), just as is the case with finite measuring numbers. We also have, for fixed $\delta$, that the (measuring) number of $\delta$ indivisibles in an interval is proportional to the length $l$ of the interval.

In this interpretation the cardinal number argument, that intervals of different lengths have the same cardinal, as in Figure 2, is easily rationalised. Over an interval with infinitesimals the map taking $x$ to $2 x$ doubles the size of a $\delta$-indivisible to a $2 \delta$-indivisible. In measuring terms we see that the number of $\delta$-indivisibles in an interval length $l$ is $l / \delta$, whilst the number of $2 \delta$-indivisibles in an interval of length $2 l$ is $(2 l) /(2 \delta)$, which is the same. If 'points' have infinitesimal size, then the only way that two intervals of differing length can have the same (measuring) number of 'points' is if the size of the points is changed in proportion to the length of the interval, which is precisely what one would expect.

\section{Non-standard integers}

The main concept missing in the infinite extension of measuring number so far described is a notion of 'infinite integer'. In the finite case we can 
count the number of closed intervals length $d$ required to cover an interval length $l$. It is the integer $n$ where

$$
n-1<l / d \leq n \text {. }
$$

In the superreals there are no 'infinite integers' which extend this idea. To get an infinite extension allowing us to blend measuring and counting we need to work in the much more comprehensive system of hyperreal numbers $\boldsymbol{R}^{*}$ discovered by Robinson [4] in the 1960's. The hyperreals $\boldsymbol{R}^{*}$ contain a subring of non-standard integers $\boldsymbol{Z} *$ such that finite elements in $Z^{*}$ are the integers $\boldsymbol{Z}$ in the usual sense. A characteristic property of $\boldsymbol{Z}^{*}$ is that given any $x \in \boldsymbol{R}^{*}$ whatsoever, there exists a unique $N \in Z^{*}$ such that

$$
N-1<x \leq N \text {. }
$$

If we wish to know how many $\delta$-infinitesimals in an interval of length 1 , then we can take the measuring number $l / \delta \in \boldsymbol{R}^{*}$ and find $N \in$ $Z^{*}$ such that

$$
N-1<l / \delta \leq N
$$

and then we can define the counting number of intervals (in a measuring sense!) to be $N$. For finite $l / \delta$, this gives the usual finite integer solution $N \in \boldsymbol{Z}$, so that the non-standard version is a natural extension of the finite concept.

There is also a very natural version of the notion of the number of $\delta$ indivisibles necessary to cover an interval

$$
[a, b]^{*}=\left\{x \in \boldsymbol{R}^{*} \mid a \leq x \leq b\right\} .
$$

We need only take the non-standard integer $N$ where

$$
N-1<(b-a) / \delta \leq N \text {. }
$$

and take

$$
I_{n}=\left\{x \in R^{*} \mid a+(n-1) \delta \leq x \leq a+n \delta\right\}
$$

for $1 \leq n \leq N$. Then each $I_{n}$ is a $\delta$-indivisible and these indivisibles (for $1 \leq n \leq N)$ precisely cover the interval $[a, b]^{*}$.

Once more the non-standard integer is a much more natural way of counting the $\delta$-indivisibles in a hyperreal interval than any cardinal interpretation. If we were to double the length of the interval, then, using

$$
N-1<(b-a) / \delta \leq N
$$

we get

$$
2 N-2<2(b-a) / \delta \leq 2 N .
$$


so the number of $\delta$-indivisibles in an interval of double length is either $2 N$ or $2 N-1$, depending on the circumstances, again reflecting the possibilities occurring in the finite case.

Were we to attempt to compute the cardinal number of $\delta$-indivisibles in a hyperreal interval, we would come up against an important technicality; there is not one version $\boldsymbol{R}^{*}$ of the hyperreals, but many, and different versions may have different cardinals (see [3]). We omit the computations in general, but note that the cardinal number of infinitesimal indivisibles in an interval depends on the cardinal of the underlying continuum but is independent of the length of the interval.

\section{Points in the plane}

Similar interesting deviations occur between cardinal and measuring number interpretations of points in the plane. A cardinal interpretation tells us that the number of points in a square is the same as the number of points in an interval. The size of the square is immaterial, but we get different infinities depending on whether the points have real or just rational coordinates.

Suppose we regard a $\delta$-indivisible in the plane to be a set $D$ such that the sets of coordinates

$$
\{x \in \mathfrak{R} \mid(x, y) \in D\},\{y \in \mathfrak{R} \mid(x, y) \in D\}
$$

are both $\delta$-indivisibles in the sense of $\S 4$. Then a $\delta$-indivisible in the plane is at most a square of side-length $\delta$. If we regard such a $\delta$ indivisible as a 'point' of infinitesimal non-zero size, we may consider how many such 'points' are required to cover a square.

In cardinal terms we get the same sort of answers as in the theorems of $\S 5$. A superreal square requires at least $\aleph$ 'points' to cover it, whilst a square containing only points with coordinates in $Q(\varepsilon)$ requires only $\aleph_{0}$. In a cardinal sense, therefore, $\delta$-indivisibles behave much the same as ordinary points in the plane; just as many are required to cover a square as there are ordinary points in the corresponding continuum, but the size of the square is irrelevant.

In a measuring sense, however, the number of $\delta$-indivisibles needed to cover a square side-length $l$ is $l / \delta^{2}$. In this sense the number depends on both $l$ and $\delta$. More interesting, if we compare the number of $\delta$ indivisibles in an interval length $l$ or a square side-length $l$, the cardinal answer is that they are the same, but the measuring number gives a ratio of $l / \delta$ to $l^{2} / \delta^{2}$. Thus, in a measuring number sense, there are infinitely 
more 'points' in a square than in an interval, to be precise the ratio is the infinite number $l / \delta$.

\section{Closing remarks on intuition}

In the previous four sections we have seen how the concept of infinite measuring number yields a quite different interpretation of infinity from that of an infinite cardinal. Such measuring numbers are elements of a field and, as such, may be added, subtracted, multiplied and divided in the usual way whilst the arithmetic of cardinals is restricted to addition and multiplication. In a number of other ways they are more intuitively satisfying (though only applicable where some form of measurement is implied, such as 'points' of specific size).

One reason why measuring numbers may seem more appealing to the intuition in some cases is that a child's first intimation of the notion of a 'point' is usually that of a mark in a physical drawing where 'points' have finite (but indeterminate) size. It is natural therefore to extend our schemas by taking smaller and smaller points leading plausibly to the notion of a point of infinitesimal size.

Formal mathematicians, however, see such a phenomenon through the distorting lens of the predominating mathematical paradigm. By posing an alternative schema of infinite measuring numbers, we may at least see that our interpretation of infinity is relative to our schema of interpretation rather than an absolute form of truth.

In judging the intuitive thought of children this is particularly important, for they do not have access to the formal schemas of mature mathematicians. The 'truth' or 'falsehood' of children's intuitions must be seen in their own context rather than through a superimposed formal schema which may misrepresent them. Different formal schemas may interpret the results quite differently, for example the notion that a longer line segment has more points in it is true in a measuring schema but false in a cardinal schema.

Apparent contradictions in intuitions may also be due to a faulty schema of interpretation. For instance it seems inconsistent that two sets can have the same number of elements and yet at the same time one has more than the other. But Cantor's set theory demonstrates that this can happen precisely when the sets are infinite. The contradiction arises from attempting to impose a framework of interpretation based on experience with finite sets. 
This illustrates a common occurrence in the development of mathematical ideas, both in the history of mathematics and the development of the individual. Working in a given context certain 'facts' arise which hold in the given context but break down when the theory is broadened. The 'fact' that a proper subset of a set has a smaller number of elements is true for finite sets but can break down in the infinite case. Other examples include the 'fact' that the operation of subtraction seems always to lead to a smaller result, which fails when negative numbers are subtracted, or the 'fact' that multiplication produces a larger value, until the introduction of proper fractions falsifies that rule also.

Such intuitions based on implied truths in a restricted context can cause serious conflicts when the context is broadened. These conflicts are all the more serious when they are subliminal, unspoken and, as a consequence, unnoticed. Suppose we regard brain activity in terms of electrical resonance in a complex circuit system whose configuration is altered by a chemical action over a period of time. Sensory inputs cause the formation of configurations which become the foundation for resonance responses for later inputs. Intuitions may thus be firmly fixed by the chemical action within the brain caused by a variety of sensory input, though they are not delineated by specific, consciously noted, sensory information.

Many researchers have commented on the child's conception of various mathematical ideas. It may be that the lasting intuitions of a child can give additional insight into the initial foundations. For instance the consonance of intuitions of infinity with infinite measuring number rather than cardinal number in some children requires an answer from those psychologists who see the child's interpretation of number only within a cardinal paradigm. Likewise the psychological investigation of the child's view of the infinitely large and the infinitely small is distorted by viewing it through the blinkers of a purely cardinal interpretation. As we have seen in this article, an alternative interpretation is possible which is consistent with certain intuitions which are rejected by a cardinal viewpoint.

It is a salutary experience to learn that sophisticated theory can actually be an impediment in the understanding of intuitive notions. 


\section{APPENDIX}

Proof of Theorem 1. Denoting the cardinal number of a set A by $|\mathrm{A}|$, clearly

$$
|\mathfrak{R}| \geq|\boldsymbol{R}|=\aleph
$$

Given

$$
\alpha=b_{m} / \varepsilon^{\mathrm{m}}+\ldots++b_{1} / \varepsilon+\sum a_{n} \varepsilon^{n}
$$

then for $N=\{1,2,3, \ldots\}$, define

$$
\begin{aligned}
\phi_{\alpha}(2 n-1) & = \begin{cases}b_{n} & (n \leq m) \\
0 & (n>m)\end{cases} \\
\phi_{\alpha}(2 n) & =\alpha_{n-1}
\end{aligned}
$$

This gives a bijective correspondence between $\mathfrak{R}$ and a subset of the set of maps from $\boldsymbol{N}$ to $\boldsymbol{R}$, hence

$$
|\mathfrak{R}| \leq \aleph_{0} .
$$

where $\aleph_{0}=|N|$.

But it is a well-known result (e.g. [5, Chapter 12]) that

$$
\aleph=2^{\aleph_{0}}
$$

and by cardinal arithmetic,

$$
\aleph^{\aleph_{0}}=\left(2^{\aleph_{0}}\right)^{\aleph_{0}}=2^{\aleph_{0}{ }^{2}}=2^{\aleph_{0}}=\aleph
$$

so

$$
|\mathfrak{R}| \leq \boldsymbol{\aleph} .
$$

Taking this together with Equation (I) proves the theorem.

\section{References}

[1] Fischbein, E.: 1978, 'Intuition and mathematical education', Osnabrücker Schriften zür Mathematik, 1, 148-176.

[2] Fischbein, E., Tirosh, D. and Hess, P.: 1979, 'The intuition of infinity', Educational Studies in Mathematics 10, 340.

[3] Keisler, H. J.: 1976, Foundations of Infinitesimal Calculus, Prindle, Weber \& Schmidt, Boston.

[41 Robinson, A.: 1966, Non-standard Analysis, North Holland, Amsterdam.

[S] Stewart, I. N. and Tall, D. O.: 1977, Foundations of Mathematics, Oxford University Press, Oxford.

[6] Tall, D. O.: 1980, 'Infinitesimals constructed algebraically and interpreted geometrically', Mathematical Education for Teachers (to appear).

[7] Tall, D. O.: 1980, 'Looking at graphs through infinitesimal microscopes, windows and telescopes', Mathematical Gazette (to appear). 